\title{
Combined Modeling of Projected Evaluation of the Regional Socio-economic Development
}

\author{
Submitted 08/01/19, 1st revision 13/02/19, 2nd revision 17/04/19, accepted 02/06/19 \\ V.V. Davnis, ${ }^{1}$ V.I. Tinyakova ${ }^{2}$, A.O. Blinov ${ }^{3}$, Yu.V. Volodin ${ }^{4}$
}

\begin{abstract}
:
Purpose: The article reveals the authors' approach to forecast the socio-economic development of a region.

Design/Methodology/Approach: The framework of this approach envisages the construction of three interrelated models: a matrix predictor, an autoregressive model, and a binary choice logit model. This approach ensures an adequate reproduction of the systemic dynamics of indicators of regional socio-economic development.

Findings: Authors' models require their implementation in the state management function of the regional economy and testing with specific examples illustrating opportunities of multidimensional economic and mathematical modeling of difficult socio-economic phenomena and processes.

Practical implications: Authors' development represents a conceptual foundation for the development of digital regional socio-economic monitoring systems providing an opportunity for a qualitative increase in the reliability and relevance of prognostic parameters.

Originality/Value: The contribution of the article is assessment of an opportunity to model the dynamics of multidimensional processes with the perspective to build a combined forecast model of the socio-economic development of the region, providing for the implementation of multivariate forecast calculations.
\end{abstract}

Keywords: Forecast, region, socio-economic development, combined modeling, discretecontinuous regression matrix model.

JEL Code: C01, C02, R11.

Paper Type: Research article in Special Issue dedicated to Russian Economy.

Section 7: Economic Development.

\footnotetext{
${ }^{l}$ Doctor of Economics, Professor, Head of the Department of Information Technologies and Mathematic Methods in Economics at the FSBFEI HE "Voronezh State University", vdavnis@mail.ru

${ }^{2}$ Doctor of Economics, Professor of the Department of Informatics and Applied Mathematics at the FSBFEI HE "Russian State Social University”, tviktoria@yandex.ru

${ }^{3} \mathrm{PhD}$ in Engineering, Associate Professor of the Department of Informatics and Applied Mathematics at the FSBFEI HE "Russian State Social University".

${ }^{4}$ PhD in Physics and Mathematics, Associate Professor of the Department of Informatics and Applied Mathematics at the FSBFEI HE "Russian State Social University".
} 


\section{Introduction}

Contemporary conditions of economic activity are distinguished primarily by the multidimensional nature of the interaction and the high level of uncertainty. The need to solve this problem, first, is dictated by the fact that at present the forecast is, in fact, the only guideline in the economic activity for both commercial and state structures. Quite naturally, the significance of this single guideline automatically increases, as any solution is currently recommended to be taken through testing preemptive expectations, their reliability, as is easy to understand, determined by the effectiveness of the economic forecasting apparatus.

The issue of the multiple-option image of the future was first raised in the paper (Davnis and Tinyakova, 2005a), in which the predictive image was defined, providing a probabilistic description of the expected options together with multivariance. This is a very useful clarification of a multivariate description of the future, according to the observations of this article. It needs to be developed for the case when each version of the future is multidimensional. The possibility of modeling the dynamics of multidimensional processes is considered. Then, the manuscript contains constructing a combined forecast model for the socio-economic development of the region providing for multiple predictive calculations.

\section{Key Ideas for Constructing a Matrix Predictor}

The main idea, in accordance with which the matrix predictor is constructed, involves the use of indirect growth rates to determine the structure of proportionality in changes in multidimensional dynamics (Davnis and Tinyakova, 2005b). Such a model should ensure the transfer of the structure of interaction of processes characterizing the dynamic state of the economic system to the future. In other words, there is a "reasonable" relationship between the predicted trajectories calculated using the matrix predictor. This relationship excludes implausible predictive options. This is a very important feature of the matrix predictor. All methods and models used in problems of forecasting multidimensional dynamics should possess it. This feature can be considered as the argument due to which a matrix predictor should be used as a basic model for developing an effective apparatus of multidimensional forecasting.

Here is a brief description of the algorithm, according to which the matrix predictor model is formed. There are the following symbols: $x_{t i}-$ the value of the $i$ index at the time; $x_{t-1 i}-$ the value of the $i$ index at the time $(t-1) ; \Delta x_{t i}-$ the value of the change (increment) of index $i$. The movement can be both positive and negative. It is assumed that the magnitude of the change in arbitrary index $i$ is formed under the influence of another index $(n-1)$. Moreover, the contributions of these indicators to the cumulative movement are of the same magnitude and equally directed. Thus, by 
virtue of the assumptions made, the movement of index $i$ can be represented by the following sum:

where $v_{i j}=\frac{1}{n-1} \Delta x_{t i} / x_{t j}$

$$
\Delta x_{\mathrm{ti}}=\sum_{j \neq i} v_{i j} x_{t j},
$$

The equation (1) together with the movement in the form of difference $\Delta x_{t i}=x_{t i}-x_{t-1 i}$ allow formulating the current value of any index $i$ in the form of:

$$
x_{\mathrm{ti}}=x_{\mathrm{t}-1 \mathrm{i}}+\sum_{j \neq i \mathrm{i}} v_{\mathrm{ij}} x_{\mathrm{t} j \mathrm{j}}, \quad i=\overline{1_{s} n} .
$$

To explain all $n$ equations (1.2), the symbols should be introduced in the matrix:

$$
\mathbf{x}_{\mathrm{t}}=\left(\begin{array}{c}
x_{\mathrm{t1}} \\
x_{\mathrm{t2}} \\
\vdots \\
x_{\mathrm{tn}}
\end{array}\right), \quad \mathbf{V}=\left(\begin{array}{cccc}
0 & v_{12} & \cdots & v_{1 n} \\
v_{21} & 0 & \cdots & v_{2 n} \\
\vdots & \vdots & \vdots & \vdots \\
v_{n 1} & v_{n 2} & \cdots & 0
\end{array}\right),
$$

using them, the following equation is formulated

$$
\mathbf{x}_{\mathrm{t}}=\mathbf{x}_{\mathrm{t}-1}+\mathrm{Vx}_{\mathrm{t}} \text {. }
$$

The final version of the matrix predictor is easily obtained from (3)

$$
\mathbf{x}_{\mathrm{t}}=(\mathrm{I}-\mathrm{V})^{-1} \mathbf{x}_{\mathrm{t}-1}
$$

Thus, the system of equations (3) describes the mechanism of growth of economic indicators, and the system of equations (4) is the mechanism of their growth.

Predicted values can be obtained if $\mathbf{x}_{\mathrm{t}-1}$ in equation (4) is replaced by $\mathbf{x}_{\mathrm{t}}$

$$
\hat{\mathbf{x}}_{\mathrm{t}+1}=(\mathrm{I}-\mathrm{V})^{-1} \mathbf{x}_{\mathrm{t}}
$$

Within the framework of the considered model, the values of the forecast evaluation depend on the current values of the predicted indicators themselves and the magnitude of the current increase. Current values are defining the expectations of the future.

\section{Regression Matrix Model}

The approach based on the combined application of the ideas of constructing a matrix predictor and econometric modeling is the most productive. The implementation of this approach involves the presentation of the value $x_{t i}$ of each indicator $i$

in the econometric form

$$
x_{t i}=x_{t-1 i}+\left(x_{t i}-x_{t-1 i j}\right), \quad i=\overline{1_{s} n}
$$




$$
x_{\mathrm{ti}}=\alpha_{\mathrm{i}}+\beta_{\mathrm{i}}\left(x_{\mathrm{ti}}-x_{\mathrm{t}-1 \mathrm{ii}}\right)+\varepsilon_{\mathrm{ti}}, \quad i=\overline{\mathbb{1}_{s} n} .
$$

Estimated regression equation (7)

$$
x_{\mathrm{ti}}=\hat{\alpha}_{\mathrm{i}}+\hat{\beta}_{\mathrm{i}}\left(x_{\mathrm{ti}}-x_{\mathrm{t}-1 \mathrm{ii}}\right), \quad \quad i=\overline{1_{s} n}
$$

can be considered as an econometric analog of expression (6) on the basis of which a model matrix predictor can be built. The increment of the predicted indicator in the framework of the econometric approach is adjusted by estimating the regression coefficient, which leads to the following equation

$$
\Delta^{\beta_{i}} x_{t i}=\hat{\beta}_{i}\left(x_{t i}-x_{t-1 i}\right), \quad i=\overline{1_{s} n}
$$

This value can be represented as the following sum:

where

$$
\Delta^{\beta_{i}} x_{t i}=\sum_{j \neq i} v_{i j}^{\beta_{i}} x_{t j i}, \quad i=\overline{1_{s} n}
$$

$$
v_{i j}^{\beta_{i}}=\frac{1}{n-1} \Delta^{\beta_{i}} x_{\mathrm{ti}} / x_{\mathrm{t} j}, \quad i_{s} j=\overline{1_{s} n} .
$$

It is clear that when using the estimated regression equation (8) in the matrix predictor model, the current value of the simulated indicator is represented as

$$
x_{\mathrm{ti}}=\alpha_{\mathrm{i}}+\sum_{j \neq i \mathrm{i}} v_{\mathrm{ij}}^{\beta_{\mathrm{i}}} x_{\mathrm{t} j}, \quad i=\overline{\mathbb{1}_{s} n} .
$$

On using symbols

$$
\mathbf{x}_{\mathrm{t}}=\left(\begin{array}{c}
x_{\mathrm{t} 1} \\
x_{\mathrm{t} 2} \\
\vdots \\
x_{\mathrm{tn}}
\end{array}\right), \quad \boldsymbol{\alpha}=\left(\begin{array}{c}
\alpha_{1} \\
\alpha_{2} \\
\vdots \\
\alpha_{n}
\end{array}\right), \quad \boldsymbol{v}_{\beta}=\left(\begin{array}{cccc}
0 & v_{12}^{\beta_{1}} & \ldots & v_{1 n}^{\beta_{1}} \\
v_{21}^{\beta_{2}} & 0 & \ldots & v_{2 n}^{\beta_{2}} \\
\vdots & \vdots & \vdots & \vdots \\
v_{n 1}^{\beta_{n}} & v_{n 2}^{\beta_{n}} & \ldots & 0
\end{array}\right),
$$

the system (6) in the vector form is written as follows:

$$
x_{t}=\alpha+V_{\beta} x_{t}
$$

The role of the previous value $x_{t-1}$ by analogy with (6) in this expression is assigned to the free term of the regression equation, which significantly limits the opportunities of the matrix predictor derived from (12)

$$
x_{\mathrm{t}}=\left(\mathrm{I}-V_{\beta}\right)^{-1} \boldsymbol{\alpha}
$$

The accuracy of such a predictor largely depends on the accuracy of the regression equations that were formulated to describe the dynamics of the system of predicted indicators. However, it is not possible to construct adequate regression equations for all indicators (Ayvazyan and Mkhitaryan, 1998). Therefore, such specification 
refining is required, which, on the one hand, would increase the accuracy of the econometric component in the regression matrix model, and on the other hand, would preserve the opportunity of implementing multivariate calculations.

\section{Autoregressive Matrix Model}

Specification refining begins from the autoregressive first-order model

$$
x_{\mathrm{ti}}=a_{0 \mathrm{i}}+a_{1 \mathrm{i}} x_{\mathrm{t}-\mathrm{ii}}+\varepsilon_{\mathrm{ti}}, \quad i=\overline{1_{s} n},
$$

where $a_{0}, a_{1}-$ estimated autoregressive equation coefficients; $\varepsilon_{\mathrm{ti}^{\mathrm{i}}}-$ a random component characterizing that part of the movement $x_{\mathrm{ti}}$, which is not explained by the corresponding changes in the past.

To provide an opportunity to build the matrix predictor, the values of the lagging variable of the autoregressive model should be introduced (14) in the form described by expression (6), i.e.

$$
x_{t-1 i}=x_{t-2 i}+\left(x_{t-1 i}-x_{t-2 i}\right), \quad i=\overline{1_{s} n} .
$$

If expression (15) is used as a lagging variable, then model (14) converts into:

$$
x_{t i}=a_{0 i}+a_{1 i} x_{t-2 i}+a_{2 i}\left(x_{t-1 i}-x_{t-2 i}\right)+\varepsilon_{t i}, \quad i=\overline{1_{s} n} .
$$

Using an estimated model

$$
\hat{x}_{\mathrm{ti}}=\hat{a}_{0 \mathrm{i}}+\hat{a}_{1 \mathrm{i}} x_{\mathrm{t}-2 \mathrm{i}}+\hat{a}_{2 \mathrm{i}}\left(x_{\mathrm{t}-1 \mathrm{i}}-x_{\mathrm{t}-2 \mathrm{i}}\right), \quad i=\overline{1_{s} n},
$$

similarly to the way it was done above, the matrix predictor is built as follows

$$
x_{\mathrm{t}}=\left(I-V_{\hat{\mathscr{C}}}\right)^{-1}\left(\hat{\boldsymbol{\alpha}}_{0}+{\widehat{\boldsymbol{\alpha}_{1}}}_{x_{\mathrm{t}-2}}\right),
$$

which differs from (3) in that the vector projected evaluation is determined considering the lagging variable.

\section{Discrete-continuous Regression Matrix Model}

The problem of multivariate forecast calculations in the framework of the econometric approach can be solved by using discrete-continuous models. Here it is necessary to determine the factor, and perhaps several factors on which the expected options for the development of the dynamics of the predicted indicators system depend. It is also needed to define the apparatus with which the idea of multivariate forecast calculations can be realized. 
It is advisable to use the gross regional product (GRP) as such a factor, and the econometric models of binary and multiple choice as an apparatus. GRP is an indicator that is formed by the entire regional system and which, in fact, most fully characterizes the results of this system activity. Naturally, it is precisely the indicator in which the options of regional development that took place in the past are reflected. Therefore, it is hoped that this relationship will continue in the future. Given this, it is proposed to introduce a discrete variable in each regression model of the predicted index, its values determined in accordance with the following rule:

$$
\begin{gathered}
z_{\mathrm{ti}}=\left\{\begin{array}{ll}
+1_{s} & e_{\mathrm{ti}} \geq 0 \\
-1, & e_{\mathrm{ti}}<0
\end{array},\right. \\
e_{\mathrm{ti}}=x_{\mathrm{ti}}-\hat{a}_{0 \mathrm{i}}-\hat{a}_{1 \mathrm{i}} x_{\mathrm{t}-2 \mathrm{i}}-\hat{a}_{2 \mathrm{i}}\left(x_{\mathrm{t}-1 \mathrm{i}}-x_{\mathrm{t}-2 \mathrm{i}}\right), \quad i=\overline{1_{s} n} .
\end{gathered}
$$

The variable introduced in this way allows rewriting (17) in the form

$$
\hat{x}_{t i}=\hat{a}_{0 i}+\hat{a}_{1 i} x_{t-2 i}+\hat{d}_{i} z_{t i}+\hat{a}_{2 i}\left(x_{t-1 i}-x_{t-2 i}\right), \quad i=\overline{1_{s} n},
$$

where the new parameter $\hat{d}_{i}$ is defined as the average value of deviations

$$
\widehat{d}_{\mathrm{i}}=\frac{1}{T} \sum_{\mathrm{t}=1}^{T}\left|e_{\mathrm{ti}}\right|, \quad \quad i=\overline{1}, \quad .
$$

To complete the construction of the model, it is necessary to envisage a mechanism in this model which would help determine what value discrete variable $z_{t i}$ should take at the pre-emptive moment of time. The solution of this question is connected with the apparatus of econometric modeling of discrete variables (Gourieroux, 2000). Using the value of the domestic regional product $r_{t}$ as a factor on which the growth or decrease of the predicted index depends, a special model can be built for this

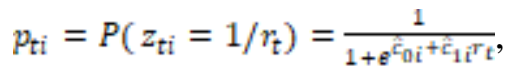

Probability $p_{\mathrm{ti}}$ calculated using this model allows replacing random variable $z_{\mathrm{ti}}$ in (21) with expectation value $E\left(z_{\mathrm{ti} i}\right)=\left(2 p_{\mathrm{ti}}-1\right)$ and using the expression in further calculations

$$
\hat{x}_{t i}=\hat{a}_{0 \mathrm{i}}+\hat{a}_{1 \mathrm{i}} x_{\mathrm{t}-2 \mathrm{i}}+\hat{d}_{\mathrm{i}}\left(2 p_{\mathrm{ti}}-1\right)+\hat{a}_{2 \mathrm{i}}\left(x_{\mathrm{t}-1 \mathrm{i}}-x_{\mathrm{t}-2 \mathrm{i}}\right), \quad i=\overline{1_{s} n} .
$$

On using symbols

$$
\hat{\boldsymbol{\alpha}}_{0}=\left(\begin{array}{c}
\hat{a}_{01} \\
\hat{a}_{02} \\
\ldots \\
\hat{a}_{0 \mathrm{n}}
\end{array}\right) \quad \hat{\boldsymbol{\alpha}}_{1} \boldsymbol{x}_{\mathrm{t}-2}=\left(\begin{array}{c}
\hat{a}_{11} x_{\mathrm{t}-21} \\
\hat{a}_{12} x_{t-22} \\
\ldots \\
\hat{a}_{1 n} x_{t-2 n}
\end{array}\right) \quad \hat{\boldsymbol{d}}\left(p_{t}\right)=\left(\begin{array}{c}
\hat{d}_{1}\left[2 p_{t 1}-1\right] \\
\hat{d}_{2}\left[2 p_{t 2}-1\right] \\
\ldots \ldots \ldots \\
\hat{d}_{n}\left[2 p_{t n}-1\right]
\end{array}\right) \text {, }
$$

the discrete-continuous regression matrix model can be written as follows: 
354

$$
x_{t}=\left(I-V_{\hat{\alpha}}\right)^{-1}\left(\hat{\alpha}_{0}+\widehat{\alpha}_{1} x_{t-2}+\tilde{d}\left(p_{t}\right)\right)
$$

In accordance with this model, the values of the future are formed from options of the past, which are selected depending on the expected situation. Expectations of the situation are determined by the forecast estimate of the GRP obtained using the forecast model. The autoregressive model is recommended to use as the forecast model.

$$
\hat{r}_{\mathrm{t}}=\hat{b}_{0}+\hat{b}_{1} x_{t-1} \text {. }
$$

It can adjust the forecast estimates of GRP by means of expert evaluation or using forecast evaluation obtained using other models. The forecast estimation of the GRP allows determining the probability of the expected increase or decrease of its values for each indicator with the help of (23). In fact, to obtain forecast estimates, a complex of three models is used.

\section{Conclusion}

The development of forecasting models for multidimensional processes describing the socio-economic development of regions can be considered a timely and very useful result. At present, when issues related to the development of the digital economy are being discussed, the lack of an adequate apparatus for describing our future in a digital format naturally emphasizes the need for such developments.

\section{References:}

Ayvasyan, S.A., Mkhitaryan, V.S. 1998. Applied Statistics and Fundamentalds of Econometrics. Moscow, YUNITI, 220.

Davnis, V.V., Tinyakova, V.I. 2005a. Forecast and an adequate image of the future. Bulletin of Voronezh State University, Economics and Management, 2, 183-190.

Davnis, V.V., Tinyakova, V.I. 2005b. Forecast Models of Expert Preferences. Voronezh, Voronezh State University, 248.

Gourieroux, Ch. 2000. Econometrics of Qualitative Dependent Variables. Cambridge, The Pitt Building, 371. 